\title{
PROFILES OF LIPASE AND PROTEASE ENZYMATIC ACTIVITIES AND ANTIMICROBIAL RESISTANCE OF SOME BACTERIAL ISOLATES RECOVERED FROM BLOOD SAMPLES \\ Amira A. Abdelbaset ${ }^{1}$, Khaled M. Aboshanab ${ }^{2}$, Mohamed M. Aboulwafa ${ }^{2}$, Hala A. Farrag $^{1}$ \\ ${ }^{1}$ Drug Radiation Research Department, National Center for Radiation Research and Technology \\ (NCRRT), Atomic Energy Authority, Cairo, Egypt. \\ ${ }^{2}$ Department of Microbiology and Immunology, Faculty of Pharmacy, Ain Shams University, Organization of African Unity St., POB: 11566, Abbassia, Cairo, Egypt .
}

\begin{abstract}
Bloodstream infection (BSI) continues to be a life threatening condition. The host response to such infection varies from clinical signs. In this study, blood samples were collected from immonocompromised patients having leukemia and immunocompetent patients during onset of fever. blood samples were collected at fever onset to predict gram negative bacteremia and study the relation between virulence factors represented as lipase and protease enzymes production in gram negative bacteremia in comparison to antibacterial resistance profiles. Twenty four (24) feverish in-patients were enrolled in the study. Blood samples were collected and cultured on blood culture media for isolation of gram negative organisms and identified by API 20E technique then antimicrobial susceptibility tests and enzymatic activity of lipase and protease were performed. Gram negative bacteria isolated from blood samples showed high resistance against most of the antibacterial agents used. Even in presence of protease and lipase activity as virulence factors. We concluded that continuous monitoring of antimicrobial resistance is required among in-patients to decrease the risk of bacteremia and resistance.
\end{abstract}




\section{Introduction}

The bloodstream was the second most frequent infection site, representing $20 \%$ of all infections (Stéphane et al., 2004). Bloodstream infection (BSI) continues to be a life threatening condition. Bacteremia refers to a bacterial invasion into blood circulation. Bacteremia may also result from any type of dental or surgical procedure. Bacteremia may cause no symptoms and resolve without treatment, or it may produce fever and other symptoms of infection depending on whether the organism was able to replicate themselves in the blood stream. For most people, the immune system should "notice" the organisms immediately and respond with specialized white blood cells to search out and destroy them. Of course, it is possible for bacteremia to progress to septicemia, especially if an individual has a weakened immune system (Young, 2012). Septicemia, sometimes called sepsis, also refers to the presence of bacteria in the blood with replication to cause an infection, but this is an infection that moves rapidly and is life-threatening (Young, 2012). Invading microorganisms induce the release of a large number of humoral and cellular proinflammatory mediators, causing systemic inflammatory response syndrome (SIRS) (Hülya et al., 2006). Nosocomial bloodstream infection (BSI) is a major complication of intensive care unit (ICU) admission. Physiological features such as fever, tachycardia and tachypnea have been proposed as indicators of sepsis. These findings may be sensitive, but are less specific in the diagnosis of systemic inflammation or infection (Dominique et al., 2007). Despite recent advances in critical care medicine, the mortality of sepsis in ICU remains high. Among various infections underlying sepsis, bacteremia is recognized as a critical condition that influences the outcome of sepsis and is reportedly associated with an attributable mortality of approximately 35\% (Ryuzo et al., 2010).

People in good health with strong immune systems rarely develop bacteremia. However, when bacteria are introduced directly into the circulatory system, especially in a person who is ill or undergoing aggressive medical treatment, the immune system may not be able to cope with the invasion and symptoms of bacteremia may develop. Symptoms of bacteremia may include: fever over $101{ }^{\circ} \mathrm{F}\left(38.3^{\circ} \mathrm{C}\right)$, chills, malaise, abdominal nausea, vomiting, diarrhea, anxiety, shortness of breath and confusion. Conditions which increase the chances of developing bacteremia include: immune suppression, either due to HIV infection or drug therapy, antibiotic therapy which changes the balance of bacterial types in the body, prolonged or severe illness ,alcoholism or other drug abuse, malnutrition, diseases or drug therapy that cause ulcers in the intestines, e.g. chemotherapy for cancer (Young, 2012). Infections are still the major cause of treatment-related morbidity and mortality in cancer patients. The malignant disease and the intensive chemotherapy may cause an impaired host defence to infection. Key factors are the intensity and duration of neutropenia, but a decreased function of granulocytes and disturbances of natural barriers may substantially add to the risk of serious infections (Miriam $\boldsymbol{e t}$ al., 2008). BSI verified by a positive blood culture is a sign of poor prognosis and predisposes patients to vascular hypotension and shock, which are associated with high mortality rates. The clinical symptoms of 
systemic inflammation associated with BSI, such as the criteria defining systemic inflammatory response syndrome (SIRS), derive from the host's innate immune response to invading organisms. This response is characterized by activation of phagocytes and systemic release of soluble mediators of inflammation (Aalto, 2004). Gram-negative bacteria play an important role in bloodstream infections about $30 \%$ of cases in the ICU are caused by one or another species of klebsiella, E. coli, enterobacter species, and $P$. aeruginosa (Michael et al., 2014). LPS in gram negative bacteria leads to increases in the expression of immune mediators (Yoshihiko et al., 2008).

The induction of inflammation by bacterial and viral infections increases cancer risk (de Martel and Franceschi, 2009).

Early recognition of BSI (Blood stream Infection) and administration of appropriate antimicrobial drugs play a crucial role in reducing mortality in communityacquired infections (Aalto, 2004). If physicians were able to rely on an early indicator of bacteremia, they could restrict their antibiotic prescriptions to the right indications, they could start therapy earlier and they could limit the number of blood samples to be obtained for culture.

\section{Materials and Methods}

Patients and samples

A total of 24 in-patients with fever onset admitted to public hospitals, private hospitals and Naser Institute hospital, Cairo, Egypt were included in this study. fifteen (15) were cancer patients and 9 were non-cancer patients. Patients on prior treatment with drugs and antibiotics were excluded from the study. Blood samples were drawn by medical staff. Blood samples were collected for bacterial culture in blood culture bottles (BACTEC Peds Plus ${ }^{\mathrm{TM}} / \mathrm{F}$, Becton Dickinson, Europe, meylan, France) by vein puncture. Contaminated blood cultures or cultures positive for microbial growth other than gram negative bacilli were excluded from the study.

\section{Bacterial strains}

In case of positive growth and after incubation for $2-7$ days at $37^{\circ} \mathrm{C}$, cultivation was carried out on blood agar plates and MacConkey's agar plates (Oxoid, England) for isolation of gram negative strains only. Identification of isolated organisms from blood specimens was done by macroscopical, microscopical and biochemical examination then identification using API 20E (BioMérieux, France).

\section{Determination of Antimicrobial susceptibility pattern:}

Some of the isolated pathogenic bacteria from blood samples were subjected to antimicrobial susceptibility tests using 14 different antimicrobial agents All of these selected (14) antimicrobial agents were supplied from (Oxoid, England). Disks as well as zones readings chart were supplied by Oxoid, England. All of these antibiotics were used as a commercially prepared discs $(6 \mathrm{~mm})$ in diameter, The content of antibiotic disks were not varied more than the limits set by the National Committee for Clinical Laboratory Standards (NCCLS 2000 and 2005). This test was done as [Kirby-Bauer 
disk susceptibility test] according to (Bauer et al., 1966) and as described by (Matsen and Barry, 1974 and Jorgensen et al., 1999)

Detection of lipase and protease enzymes production:

It was performed using Tween-agar plates (Thaler et al., 1997) and Gelatin-agar plates (Collee et al., 1996) respectively.

\section{Statistical methods}

The significance of differences between groups was calculated with t-test and statistical significance was designated at the 95\% confidence level (two-sided $P$ ).

\section{Results}

The numbers of different bacterial species and their relative percentages isolated from 24 patients are represented in table 1.

Table (1): Number and frequency of bacterial strains isolated from cancer and noncancer patients

\begin{tabular}{|c|c|c|}
\hline Bacterial strains & Cancer patients $(\mathrm{n}=15)$ & Non-cancer patients(n=9) \\
\hline Escherichia coli & $(41 \%)$ & $(38 \%)$ \\
\hline Klebsiella pneumoniae & $(23 \%)$ & $(41 \%)$ \\
\hline Pseudomonas fluorescence & $(6 \%)$ & $(7 \%)$ \\
\hline Pseudomonas putida & $(12 \%)$ & - \\
\hline Pseudomonas aeruginosa & $(9 \%)$ & $(7 \%)$ \\
\hline Acinetobacter baumnanii & $(9 \%)$ & \\
\hline
\end{tabular}

Antimicrobial susceptibility of some bacterial isolates recovered from patients:

The susceptibilities of the tested isolates against different antimicrobial agents were determined and the results are shown in tables 2. 
Table (2):Antibacterial susceptibilities of some selected bacterial isolates recovered from patients against different antimicrobial agents

\begin{tabular}{|c|c|c|c|c|c|c|c|c|c|c|c|c|c|c|c|}
\hline \multirow{2}{*}{$\begin{array}{l}\text { Patie } \\
\text { nt's } \\
\text { no. }\end{array}$} & \multirow{2}{*}{$\begin{array}{c}\text { Recovered } \\
\text { bacterial } \\
\text { isolate }\end{array}$} & \multicolumn{14}{|c|}{ Mean inhibition zone diameter $(\mathrm{mm}) /$ Susceptibility profile against ${ }^{*}$} \\
\hline & & $\begin{array}{l}\mathrm{A} \\
\mathrm{K}\end{array}$ & $\begin{array}{c}\mathrm{SA} \\
\mathrm{M}\end{array}$ & $\begin{array}{c}\mathrm{FE} \\
\mathrm{P}\end{array}$ & $\begin{array}{c}\text { CT } \\
\mathrm{X}\end{array}$ & $\begin{array}{c}\mathrm{C} \\
\mathrm{AZ}\end{array}$ & $\mathrm{C}$ & $\begin{array}{l}\mathrm{A} \\
\mathrm{M} \\
\mathrm{C}\end{array}$ & $\begin{array}{l}\mathrm{C} \\
\mathrm{N}\end{array}$ & $\begin{array}{l}\text { IP } \\
\mathrm{M}\end{array}$ & OFX & SXT & TOB & $\begin{array}{c}\text { TZ } \\
\text { B }\end{array}$ & $\begin{array}{c}\text { LE } \\
V\end{array}$ \\
\hline 1 & E. coli & $\begin{array}{c}18 / \\
S\end{array}$ & $\begin{array}{l}9 / \\
\mathrm{R}\end{array}$ & $\begin{array}{c}12 / \\
\mathrm{R}\end{array}$ & $\begin{array}{l}6 / \\
\mathrm{R}\end{array}$ & $\begin{array}{c}10 / \\
\mathrm{R}\end{array}$ & $\begin{array}{c}20 / \\
S\end{array}$ & $\begin{array}{c}12 / \\
\mathrm{R}\end{array}$ & $\begin{array}{l}6 / \\
\mathrm{R}\end{array}$ & $\begin{array}{c}26 / \\
S\end{array}$ & $6 / \mathrm{R}$ & $6 / \mathrm{R}$ & $6 / \mathrm{R}$ & $\begin{array}{l}20 / \\
\text { IR }\end{array}$ & $\begin{array}{l}6 / \\
\mathrm{R}\end{array}$ \\
\hline 2 & E. coli & $\begin{array}{c}17 / \\
S\end{array}$ & $\begin{array}{l}6 / \\
\mathrm{R}\end{array}$ & $\begin{array}{c}28 / \\
S\end{array}$ & $\begin{array}{c}23 / \\
\text { S }\end{array}$ & $\begin{array}{c}21 / \\
S\end{array}$ & $\begin{array}{l}6 / \\
\mathrm{R}\end{array}$ & $\begin{array}{l}15 / \\
\text { IR }\end{array}$ & $\begin{array}{c}15 / \\
S\end{array}$ & $\begin{array}{c}28 / \\
S\end{array}$ & $6 / \mathrm{R}$ & $6 / \mathrm{R}$ & $15 / \mathrm{I}$ & $\begin{array}{l}20 / \\
\text { IS }\end{array}$ & $\begin{array}{l}6 / \\
\mathrm{R}\end{array}$ \\
\hline 3 & E. coli & $\begin{array}{c}21 / \\
\mathrm{R}\end{array}$ & $\begin{array}{l}12 / \\
\text { IR }\end{array}$ & $\begin{array}{c}18 / \\
\text { S }\end{array}$ & $\begin{array}{c}12 / \\
\mathrm{R}\end{array}$ & $\begin{array}{c}18 / \\
\mathrm{S}\end{array}$ & $\begin{array}{c}22 / \\
\mathrm{S}\end{array}$ & $\begin{array}{l}6 / \\
\mathrm{R}\end{array}$ & $\begin{array}{c}11 / \\
\mathrm{R}\end{array}$ & $\begin{array}{c}26 / \\
S\end{array}$ & $31 / \mathrm{S}$ & $\begin{array}{c}11 / \mathrm{I} \\
\mathrm{R}\end{array}$ & $6 / \mathrm{R}$ & $\begin{array}{l}9 / \\
\mathrm{R}\end{array}$ & $\begin{array}{l}6 / \\
\mathrm{R}\end{array}$ \\
\hline 4 & E .coli & $\begin{array}{c}22 / \\
\mathrm{S}\end{array}$ & $\begin{array}{l}8 / \\
\mathrm{R}\end{array}$ & $\begin{array}{c}26 / \\
S\end{array}$ & $\begin{array}{c}29 / \\
\text { S }\end{array}$ & $\begin{array}{c}22 / \\
\mathrm{S}\end{array}$ & $\begin{array}{c}25 / \\
S\end{array}$ & $\begin{array}{c}15 / \\
\mathrm{I}\end{array}$ & $\begin{array}{c}20 / \\
S\end{array}$ & $\begin{array}{c}26 / \\
S\end{array}$ & $27 / S$ & $6 / \mathrm{R}$ & $22 / \mathrm{S}$ & $\begin{array}{c}25 / \\
\mathrm{S}\end{array}$ & $\begin{array}{c}30 / \\
\mathrm{S}\end{array}$ \\
\hline 5 & E. coli & $\begin{array}{c}17 / \\
S\end{array}$ & $\begin{array}{l}8 / \\
\mathrm{R}\end{array}$ & $\begin{array}{c}13 / \\
\mathrm{R}\end{array}$ & $\begin{array}{l}8 / \\
\mathrm{R}\end{array}$ & $\begin{array}{c}12 / \\
\mathrm{R}\end{array}$ & $\begin{array}{c}23 / \\
\mathrm{S}\end{array}$ & $\begin{array}{l}14 / \\
\text { IR }\end{array}$ & $\begin{array}{l}6 / \\
\mathrm{R}\end{array}$ & $\begin{array}{c}28 / \\
\mathrm{S}\end{array}$ & $6 / \mathrm{R}$ & $6 / \mathrm{R}$ & 9/R & $\begin{array}{c}21 / \\
\mathrm{S}\end{array}$ & $\begin{array}{l}8 / \\
\mathrm{R}\end{array}$ \\
\hline 6 & E. coli & $\begin{array}{c}20 / \\
\mathrm{S}\end{array}$ & $\begin{array}{l}9 / \\
\mathrm{R}\end{array}$ & $\begin{array}{c}29 / \\
S\end{array}$ & $\begin{array}{c}25 / \\
S\end{array}$ & $\begin{array}{c}20 / \\
\mathrm{S}\end{array}$ & $\begin{array}{l}14 / \\
\text { IR }\end{array}$ & $\begin{array}{c}18 / \\
S\end{array}$ & $\begin{array}{c}21 / \\
S\end{array}$ & $\begin{array}{c}28 / \\
S\end{array}$ & $6 / \mathrm{R}$ & $6 / \mathrm{R}$ & $20 / \mathrm{S}$ & $\begin{array}{c}22 / \\
\mathrm{S}\end{array}$ & $\begin{array}{l}6 / \\
\mathrm{R}\end{array}$ \\
\hline 7 & $\begin{array}{c}\mathrm{K} . \\
\text { pneumonia } \\
\mathrm{e}\end{array}$ & $\begin{array}{c}18 / \\
\mathrm{R}\end{array}$ & $\begin{array}{l}\text { 9/ } \\
\mathrm{R}\end{array}$ & $\begin{array}{c}22 / \\
\mathrm{S}\end{array}$ & $\begin{array}{c}18 / \\
\text { I }\end{array}$ & $\begin{array}{c}10 / \\
\mathrm{R}\end{array}$ & $\begin{array}{l}\text { 9/ } \\
\mathrm{R}\end{array}$ & $\begin{array}{l}15 / \\
\text { IR }\end{array}$ & $\begin{array}{c}10 / \\
\mathrm{R}\end{array}$ & $\begin{array}{c}25 / \\
S\end{array}$ & $6 / \mathrm{R}$ & $6 / \mathrm{R}$ & $11 / \mathrm{R}$ & $\begin{array}{c}21 / \\
S\end{array}$ & $\begin{array}{l}\text { 9/ } \\
\mathrm{R}\end{array}$ \\
\hline 8 & $\begin{array}{c}\mathrm{K} . \\
\text { pneumonia } \\
\text { ee }\end{array}$ & $\begin{array}{c}14 / \\
\text { I }\end{array}$ & $\begin{array}{l}6 / \\
\mathrm{R}\end{array}$ & $\begin{array}{l}6 / \\
\mathrm{R}\end{array}$ & $\begin{array}{l}6 / \\
\mathrm{R}\end{array}$ & $\begin{array}{l}6 / \\
\mathrm{R}\end{array}$ & $\begin{array}{c}18 / \\
\text { S }\end{array}$ & $\begin{array}{l}6 / \\
\mathrm{R}\end{array}$ & $\begin{array}{l}6 / \\
\mathrm{R}\end{array}$ & $\begin{array}{c}18 / \\
\text { S }\end{array}$ & $6 / \mathrm{R}$ & $\begin{array}{c}11 / \mathrm{I} \\
\mathrm{R}\end{array}$ & $6 / \mathrm{R}$ & $\begin{array}{l}9 / \\
\mathrm{R}\end{array}$ & $\begin{array}{l}6 / \\
\mathrm{R}\end{array}$ \\
\hline 9 & $\begin{array}{c}\mathrm{K} . \\
\text { pneumonia } \\
\mathrm{e}\end{array}$ & $\begin{array}{c}17 / \\
S\end{array}$ & $\begin{array}{l}6 / \\
R\end{array}$ & $\begin{array}{c}12 / \\
\mathrm{R}\end{array}$ & $\begin{array}{l}6 / \\
R\end{array}$ & $\begin{array}{c}18 / \\
S\end{array}$ & $\begin{array}{c}25 / \\
S\end{array}$ & $\begin{array}{c}11 / \\
\mathrm{R}\end{array}$ & $\begin{array}{l}6 / \\
R\end{array}$ & $\begin{array}{c}25 / \\
S\end{array}$ & $18 / \mathrm{S}$ & $6 / \mathrm{R}$ & $6 / \mathrm{R}$ & $\begin{array}{l}20 / \\
\text { IS }\end{array}$ & $\begin{array}{c}21 / \\
S\end{array}$ \\
\hline 10 & $\begin{array}{c}\mathrm{K} . \\
\text { pneumonia } \\
\mathrm{e}\end{array}$ & $\begin{array}{c}19 / \\
S\end{array}$ & $\begin{array}{l}6 / \\
\mathrm{R}\end{array}$ & $\begin{array}{c}27 / \\
S\end{array}$ & $\begin{array}{l}\text { 16/ } \\
\text { IR }\end{array}$ & $\begin{array}{c}20 / \\
S\end{array}$ & $\begin{array}{c}20 / \\
\mathrm{S}\end{array}$ & $\begin{array}{l}6 / \\
\mathrm{R}\end{array}$ & $\begin{array}{l}8 / \\
R\end{array}$ & $\begin{array}{c}18 / \\
S\end{array}$ & $6 / \mathrm{R}$ & 9/R & $10 / \mathrm{R}$ & $\begin{array}{l}9 / \\
\mathrm{R}\end{array}$ & $\begin{array}{l}8 / \\
\mathrm{R}\end{array}$ \\
\hline 11 & $\begin{array}{c}\mathrm{P} . \\
\text { fluorescen } \\
\text { ce } \\
\end{array}$ & $\begin{array}{c}19 / \\
S\end{array}$ & $\begin{array}{l}6 / \\
R\end{array}$ & $\begin{array}{c}21 / \\
S\end{array}$ & $\begin{array}{c}10 / \\
\mathrm{R}\end{array}$ & $\begin{array}{c}16 / \\
\text { I }\end{array}$ & $\begin{array}{c}27 / \\
S\end{array}$ & $\begin{array}{l}6 / \\
R\end{array}$ & $\begin{array}{c}12 / \\
\mathrm{R}\end{array}$ & $\begin{array}{l}17 / \\
\text { IS }\end{array}$ & $30 / \mathrm{S}$ & $6 / \mathrm{R}$ & $12 / \mathrm{R}$ & $\begin{array}{c}15 / \\
\mathrm{R}\end{array}$ & $\begin{array}{c}32 / \\
\mathrm{S}\end{array}$ \\
\hline 12 & $\begin{array}{c}\mathrm{P} . \\
\text { aeruginosa }\end{array}$ & $\begin{array}{c}25 / \\
\mathrm{S}\end{array}$ & $\begin{array}{l}6 / \\
\mathrm{R}\end{array}$ & $\begin{array}{c}27 / \\
S\end{array}$ & $\begin{array}{l}16 / \\
\text { IR }\end{array}$ & $\begin{array}{c}25 / \\
\mathrm{S}\end{array}$ & $\begin{array}{l}6 / \\
\mathrm{R}\end{array}$ & $\begin{array}{l}6 / \\
\mathrm{R} \\
\end{array}$ & $\begin{array}{c}18 / \\
\mathrm{S}\end{array}$ & $\begin{array}{c}30 / \\
\mathrm{S}\end{array}$ & $20 / \mathrm{S}$ & $\begin{array}{c}15 / \mathrm{I} \\
\mathrm{R}\end{array}$ & $23 / \mathrm{S}$ & $\begin{array}{c}31 / \\
\mathrm{S}\end{array}$ & $\begin{array}{c}20 / \\
\mathrm{S}\end{array}$ \\
\hline 13 & p. putida & $\begin{array}{l}6 / \\
\mathrm{R}\end{array}$ & $\begin{array}{l}14 / \\
\text { IS }\end{array}$ & $\begin{array}{l}6 / \\
\mathrm{R}\end{array}$ & $\begin{array}{l}6 / \\
\mathrm{R}\end{array}$ & $\begin{array}{l}6 / \\
\mathrm{R}\end{array}$ & $\begin{array}{l}9 / \\
\mathrm{R}\end{array}$ & $\begin{array}{l}6 / \\
\mathrm{R}\end{array}$ & $\begin{array}{c}12 / \\
\mathrm{R}\end{array}$ & $\begin{array}{l}15 / \\
\text { IR }\end{array}$ & $6 / \mathrm{R}$ & $6 / \mathrm{R}$ & $11 / \mathrm{R}$ & $\begin{array}{c}15 / \\
\mathrm{R}\end{array}$ & $\begin{array}{c}10 / \\
\mathrm{R}\end{array}$ \\
\hline 14 & $\begin{array}{c}\text { A. } \\
\text { baumennii }\end{array}$ & $\begin{array}{l}6 / \\
\mathrm{R}\end{array}$ & $\begin{array}{c}17 / \\
S\end{array}$ & $\begin{array}{l}6 / \\
\mathrm{R} \\
\end{array}$ & $\begin{array}{l}6 / \\
\mathrm{R}\end{array}$ & $\begin{array}{l}6 / \\
\mathrm{R}\end{array}$ & $\begin{array}{l}6 / \\
\mathrm{R} \\
\end{array}$ & $\begin{array}{l}6 / \\
\mathrm{R} \\
\end{array}$ & $\begin{array}{l}6 / \\
\mathrm{R}\end{array}$ & $\begin{array}{l}17 / \\
\text { IS }\end{array}$ & $6 / \mathrm{R}$ & $6 / \mathrm{R}$ & $6 / \mathrm{R}$ & $\begin{array}{c}16 / \\
S\end{array}$ & $\begin{array}{l}8 / \\
\mathrm{R}\end{array}$ \\
\hline 15 & $\begin{array}{c}\text { A. } \\
\text { baumennii }\end{array}$ & $\begin{array}{l}6 / \\
\mathrm{R}\end{array}$ & $\begin{array}{c}11 / \\
\mathrm{R}\end{array}$ & $\begin{array}{l}6 / \\
\mathrm{R}\end{array}$ & $\begin{array}{l}6 / \\
\mathrm{R}\end{array}$ & $\begin{array}{l}6 / \\
\mathrm{R}\end{array}$ & $\begin{array}{l}6 / \\
\mathrm{R}\end{array}$ & $\begin{array}{l}6 / \\
\mathrm{R} \\
\end{array}$ & $\begin{array}{c}12 / \\
\mathrm{R}\end{array}$ & $\begin{array}{l}\text { 14/ } \\
\text { IR }\end{array}$ & $6 / \mathrm{R}$ & $6 / \mathrm{R}$ & $12 / \mathrm{R}$ & $\begin{array}{l}6 / \\
\mathrm{R}\end{array}$ & $\begin{array}{l}6 / \\
\mathrm{R}\end{array}$ \\
\hline 16 & E. coli & $\begin{array}{l}16 / \\
\text { IS }\end{array}$ & $\begin{array}{l}6 / \\
\mathrm{R} \\
\end{array}$ & $\begin{array}{l}6 / \\
\mathrm{R} \\
\end{array}$ & $\begin{array}{l}6 / \\
\mathrm{R}\end{array}$ & $\begin{array}{l}6 / \\
\mathrm{R}\end{array}$ & $\begin{array}{c}20 / \\
\mathrm{S}\end{array}$ & $\begin{array}{l}8 / \\
\mathrm{R}\end{array}$ & $\begin{array}{l}9 / \\
\mathrm{R}\end{array}$ & $\begin{array}{c}24 / \\
\mathrm{S}\end{array}$ & $6 / \mathrm{R}$ & $6 / \mathrm{R}$ & $6 / \mathrm{R}$ & $\begin{array}{c}17 / \\
\mathrm{R}\end{array}$ & $\begin{array}{l}6 / \\
\mathrm{R}\end{array}$ \\
\hline 17 & E. coli & $\begin{array}{l}15 / \\
\text { IR }\end{array}$ & $\begin{array}{l}9 / \\
\mathrm{R} \\
\end{array}$ & $\begin{array}{c}12 / \\
\mathrm{R}\end{array}$ & $\begin{array}{l}6 / \\
\mathrm{R}\end{array}$ & $\begin{array}{c}12 / \\
\mathrm{R}\end{array}$ & $\begin{array}{l}6 / \\
\mathrm{R} \\
\end{array}$ & $\begin{array}{l}16 / \\
\text { IR }\end{array}$ & $\begin{array}{c}10 / \\
\mathrm{R}\end{array}$ & $\begin{array}{c}26 / \\
S\end{array}$ & $6 / \mathrm{R}$ & $6 / \mathrm{R}$ & $\begin{array}{c}14 / \mathrm{I} \\
\mathrm{S}\end{array}$ & $\begin{array}{l}20 / \\
\text { IS }\end{array}$ & $\begin{array}{l}6 / \\
\mathrm{R} \\
\end{array}$ \\
\hline
\end{tabular}




\begin{tabular}{|c|c|c|c|c|c|c|c|c|c|c|c|c|c|c|c|}
\hline 18 & E. coli & $\begin{array}{l}16 / \\
\text { IS }\end{array}$ & $\begin{array}{l}9 / \\
\mathrm{R}\end{array}$ & $\begin{array}{c}22 / \\
\mathrm{S}\end{array}$ & $\begin{array}{l}20 / \\
\text { IS }\end{array}$ & $\begin{array}{c}19 / \\
\mathrm{S}\end{array}$ & $\begin{array}{c}25 / \\
S\end{array}$ & $\begin{array}{c}12 / \\
\mathrm{R}\end{array}$ & $\begin{array}{l}6 / \\
\mathrm{R}\end{array}$ & $\begin{array}{c}29 / \\
\mathrm{S}\end{array}$ & $6 / \mathrm{R}$ & $6 / \mathrm{R}$ & $9 / \mathrm{R}$ & $\begin{array}{c}21 / \\
\mathrm{S}\end{array}$ & $\begin{array}{l}9 / \\
\mathrm{R}\end{array}$ \\
\hline 19 & $\begin{array}{c}\mathrm{K} . \\
\text { pneumonia } \\
\mathrm{e}\end{array}$ & $\begin{array}{l}15 / \\
\text { IR }\end{array}$ & $\begin{array}{l}6 / \\
\mathrm{R}\end{array}$ & $\begin{array}{l}6 / \\
\mathrm{R}\end{array}$ & $\begin{array}{l}6 / \\
\mathrm{R}\end{array}$ & $\begin{array}{l}6 / \\
\mathrm{R}\end{array}$ & $\begin{array}{c}25 / \\
S\end{array}$ & $\begin{array}{l}6 / \\
\mathrm{R}\end{array}$ & $\begin{array}{c}20 / \\
\mathrm{S}\end{array}$ & $\begin{array}{c}10 / \\
\mathrm{R}\end{array}$ & $6 / \mathrm{R}$ & $6 / \mathrm{R}$ & $11 / \mathrm{R}$ & $\begin{array}{c}9 / \mathrm{I} \\
\mathrm{R}\end{array}$ & $\begin{array}{l}6 / \\
\mathrm{R}\end{array}$ \\
\hline 20 & $\begin{array}{c}\mathrm{K} . \\
\text { pneumonia } \\
\mathrm{e}\end{array}$ & $\begin{array}{c}12 / \\
\mathrm{R}\end{array}$ & $\begin{array}{l}6 / \\
\mathrm{R}\end{array}$ & $\begin{array}{c}10 / \\
\mathrm{R}\end{array}$ & $\begin{array}{l}6 / \\
\mathrm{R}\end{array}$ & $\begin{array}{l}6 / \\
\mathrm{R}\end{array}$ & $\begin{array}{c}21 / \\
S\end{array}$ & $\begin{array}{l}6 / \\
\mathrm{R}\end{array}$ & $\begin{array}{c}16 / \\
S\end{array}$ & $\begin{array}{c}10 / \\
\mathrm{R}\end{array}$ & $16 / \mathrm{S}$ & $20 / \mathrm{S}$ & $9 / \mathrm{R}$ & $\begin{array}{l}9 / \\
\mathrm{R}\end{array}$ & $\begin{array}{c}15 / \\
\mathrm{I}\end{array}$ \\
\hline 21 & $\begin{array}{c}\mathrm{K} . \\
\text { pneumonia } \\
\mathrm{e}\end{array}$ & $\begin{array}{c}17 / \\
S\end{array}$ & $\begin{array}{l}\text { 6/ } \\
\mathrm{R}\end{array}$ & $\begin{array}{l}8 / \\
\mathrm{R}\end{array}$ & $\begin{array}{l}6 / \\
\mathrm{R}\end{array}$ & $\begin{array}{l}8 / \\
\mathrm{R}\end{array}$ & $\begin{array}{c}20 / \\
S\end{array}$ & $\begin{array}{l}9 / \\
\mathrm{R}\end{array}$ & $\begin{array}{c}21 / \\
\mathrm{S}\end{array}$ & $\begin{array}{c}25 / \\
S\end{array}$ & $6 / \mathrm{R}$ & $\begin{array}{c}15 / \mathrm{I} \\
\mathrm{S}\end{array}$ & $8 / \mathrm{R}$ & $\begin{array}{c}11 / \\
\mathrm{R}\end{array}$ & $\begin{array}{l}9 / \\
\mathrm{R}\end{array}$ \\
\hline 22 & $\begin{array}{c}\mathrm{K} . \\
\text { pneumonia } \\
\mathrm{e}\end{array}$ & $\begin{array}{l}16 / \\
\text { IS }\end{array}$ & $\begin{array}{l}6 / \\
\mathrm{R}\end{array}$ & $\begin{array}{c}14 / \\
\mathrm{R}\end{array}$ & $\begin{array}{l}8 / \\
\mathrm{R}\end{array}$ & $\begin{array}{l}6 / \\
\mathrm{R}\end{array}$ & $\begin{array}{l}6 / \\
\mathrm{R}\end{array}$ & $\begin{array}{c}11 / \\
\mathrm{R}\end{array}$ & $\begin{array}{l}12 / \\
\mathrm{R}\end{array}$ & $\begin{array}{c}25 / \\
S\end{array}$ & $18 / \mathrm{S}$ & $6 / \mathrm{R}$ & $12 / \mathrm{R}$ & $\begin{array}{c}15 / \\
\mathrm{R}\end{array}$ & $\begin{array}{c}21 / \\
\mathrm{S}\end{array}$ \\
\hline 23 & $\begin{array}{c}\mathrm{P} . \\
\text { fluorescen } \\
\text { ce }\end{array}$ & $\begin{array}{c}24 / \\
S\end{array}$ & $\begin{array}{l}6 / \\
\mathrm{R}\end{array}$ & $\begin{array}{c}20 / \\
S\end{array}$ & $\begin{array}{l}6 / \\
\mathrm{R}\end{array}$ & $\begin{array}{l}12 / \\
\mathrm{R}\end{array}$ & $\begin{array}{l}6 / \\
\mathrm{R}\end{array}$ & $\begin{array}{l}6 / \\
\mathrm{R}\end{array}$ & $\begin{array}{l}6 / \\
\mathrm{R}\end{array}$ & $\begin{array}{c}12 / \\
\mathrm{R}\end{array}$ & $\begin{array}{c}13 / \mathrm{I} \\
\mathrm{R}\end{array}$ & $6 / \mathrm{R}$ & $15 / \mathrm{S}$ & $\begin{array}{c}12 / \\
\mathrm{R}\end{array}$ & $\begin{array}{c}17 / \\
S\end{array}$ \\
\hline 24 & $\begin{array}{c}\text { A. } \\
\text { baumannii }\end{array}$ & $\begin{array}{l}6 / \\
\mathrm{R}\end{array}$ & $\begin{array}{c}11 / \\
\mathrm{R}\end{array}$ & $\begin{array}{c}13 / \\
\mathrm{R}\end{array}$ & $\begin{array}{l}6 / \\
\mathrm{R}\end{array}$ & $\begin{array}{l}6 / \\
\mathrm{R}\end{array}$ & $\begin{array}{c}15 / \\
\text { I }\end{array}$ & $\begin{array}{l}6 / \\
\mathrm{R}\end{array}$ & $\begin{array}{c}15 / \\
S\end{array}$ & $\begin{array}{l}17 / \\
\text { IS }\end{array}$ & $6 / \mathrm{R}$ & $6 / \mathrm{R}$ & $17 / S$ & $\begin{array}{c}16 / \\
\mathrm{R}\end{array}$ & $\begin{array}{l}8 / \\
\mathrm{R}\end{array}$ \\
\hline
\end{tabular}

Susceptibility profile was interpreted as $\mathrm{R}$ (Resistant), IR (Intermediate resistant) and S (Sensitive) according to table 3 recommended by NCCLS 2011

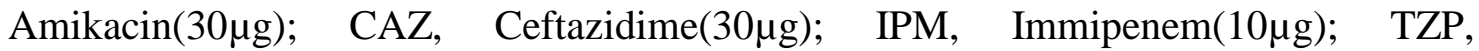
Tazobactam/Piperacillin $\quad(10 \mu \mathrm{g}) ; \quad$ SAM, Ampicillin/Sulbactam $(20 \mu \mathrm{g}) ; \quad \mathrm{C}$, Chloramphenicol $(30 \mu \mathrm{g}) ; \quad$ OFX, Ofloxacin $(5 \mu \mathrm{g}) ; \quad$ LEV, Levofloxacin $(5 \mu \mathrm{g}) ; \mathrm{FEP}$, Cefepime $(30 \mu \mathrm{g})$; AMC, Amoxycillin/Clavulanic acid(30 $\mu \mathrm{g})$; SXT, Sulphamethoxazole/Trimethoprim $(25 \mu \mathrm{g}) ; \quad$ CTX, Cefotaxime $(30 \mu \mathrm{g}) ; \quad$ CN, Gentamycin $(120 \mu \mathrm{g}) ;$ TOB, Tobramycin $(10 \mu \mathrm{g})$

The frequency percentages of resistant and susceptible bacterial isolates against different antimicrobial agents which are classified according to mechanism of action are presented in fig. 1-3. Fig. 1which showed the results of 7 tested antimicrobial agents acting on the inhibition of cell wall synthesis .It is clear that, the highest resistance frequency percentage for $\beta$ - lactam antibiotics was observed with: Ampicillin/Sulbactam (SAM) 80\%, Amoxicillin/Clavulanic acid (AMC) $66.66 \%$, Cefotaxime (CTX) 60\%, then Cefepime (FEP) 46.66\%, Ceftazidime (CAZ) 46.66\%, Tazobactam/Piperacillin (TZP) 40\%. On the other hand, Imipenem (IPM) showed no resistant phenotype for the tested isolates but intermediate resistant reaction $26.66 \%$. Some other antibiotics play an important role in the inhibition of protein synthesis, fig.2, showed that most of the tested strains were resistant to Gentamycin $(\mathrm{CN})$ and Tobramycin (TOB) $73.33 \%$ followed by $33.33 \%$ were resistant to Amikacin (AK), all previous antibiotics belonged to aminoglycosides group. Meanwhile, resistance to Chloramphenicol (C) was represented by $40 \%$. In case of antibiotics which act on inhibition of nucleic acid synthesis, the results in fig. 3 showed a high percentage of resistance among the isolated gram negative bacilli against Levofloxacin (LEV) $73.33 \%$ 
then Ofloxacin (OFX) 66.66\%. Sulphamethoxazole/Trimethoprim (SXT) as a type of antibiotic acts on inhibition of folic acid synthesis $80 \%$ of the isolates were resistant and $20 \%$ were intermediately resistant to it .Also, there was significant p-value $=0.0086$, 0.0198 and 0.05 for AMC in E. coli strains, for TOB in Pseudomonas strains and SAM in Acinetobacter strains respectively.

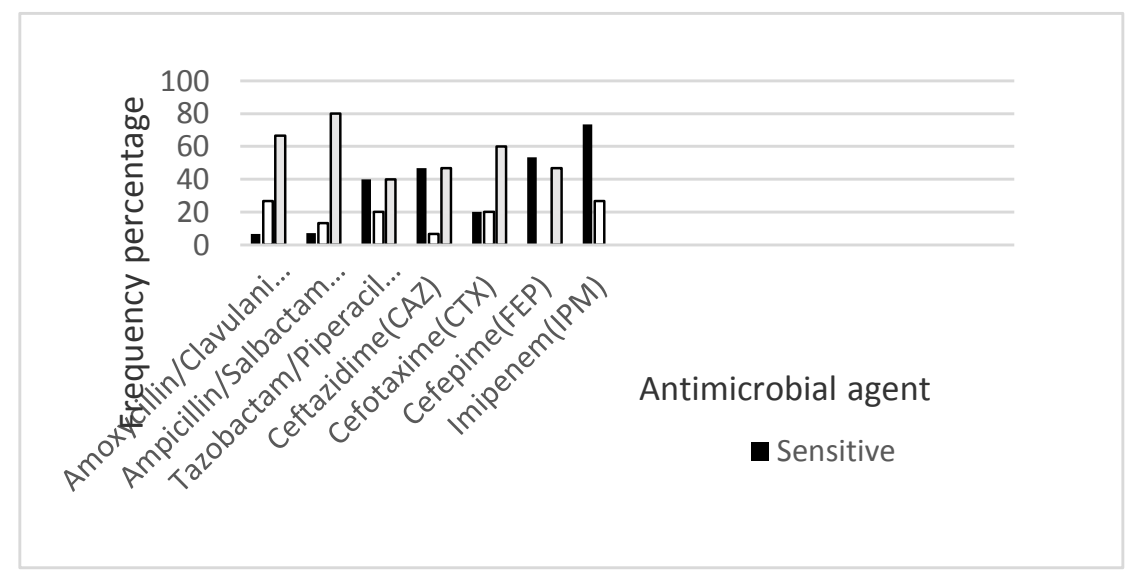

Fig.(1): Frequency percentages of different susceptibility profiles of bacterial isolates recovered from immunocompromised patients against some antimicrobial agents inhibiting bacterial cell wall synthesis

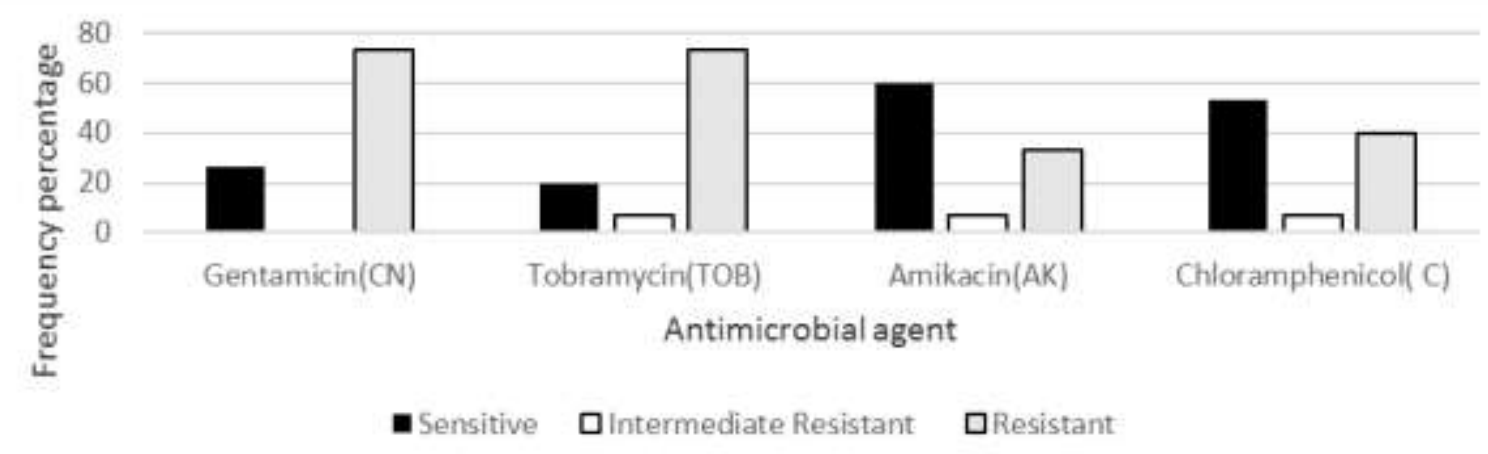

Fig.(2): Frequency percentages of different susceptibility profiles of bacterial isolates recovered from immunocompromised patients against some antimicrobial agents inhibiting protein synthesis in bacteria 


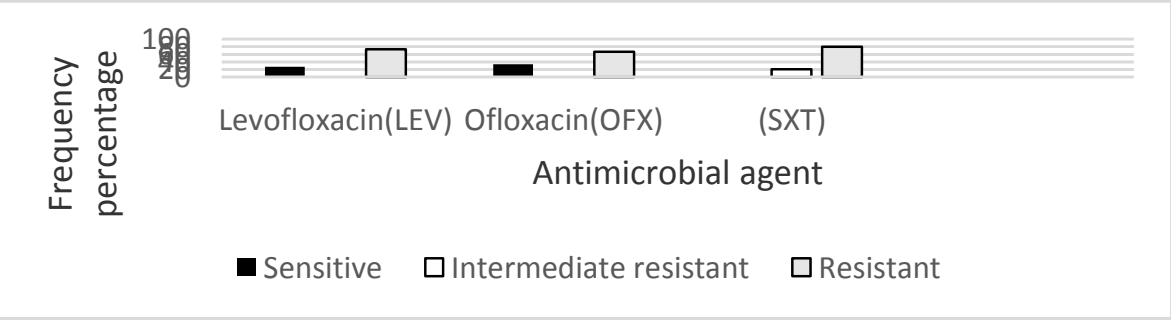

Fig.(3): Frequency percentages of different susceptibility profiles of bacterial isolates recovered from imunocompromised patients against some antimicrobial agents inhibiting nucleic and folic acids syntheses

Among the bacterial isolates recovered from immunocompetent patients, Nine (9) isolates were selected for antimicrobial susceptibility test before and after exposure to gamma irradiation. The frequency of resistant and susceptible bacterial isolates against different antibiotics by mode of action is shown in fig.4-6. In fig. 4 which showed the results of 7 antibiotics acting on the inhibition of cell wall synthesis, it was clear that ,the highest percentage of antibiotic resistance of nine bacterial strains was against Ampicillin/Sulbactam (SAM) 100\%,Amoxicillin/Clavulanic acid (AMC) 88.88\%, Cefotaxime (CTX) 88.88\%, Ceftazidime (CAZ) $88.88 \%$ then Cefepime (FEP) 77.77\%, Tazobactam/Piperacillin (TZP) 66.66\%, finally Imipenem (IPM) showed resistant reaction $33.33 \%$. Some other antibiotics play important role in the inhibition of protein synthesis.It is clear from fig.5 that most of the isolates were resistant to Tobramycin (TOB) $66.66 \%$, Gentamycin $(\mathrm{CN}) 55.55 \%$, and $22.22 \%$ were resistant to Amikacin (AK), all previous antibiotics belonged to aminoglycosides group. Resistance to Chloramphenicol (C) was represented by $33.33 \%$. In case of antibiotics which act on inhibition of nucleic acid synthesis, the results in fig.6 showed a high percentage of resistance among the tested strains against Levofloxacin (LEV) and Ofloxacin (OFX) 66.66\%. Sulphamethoxazole/Trimethoprim (SXT) as a type of antibiotic acts on inhibition of folic acid synthesis $77.77 \%$ of the treated isolates were resistant and $11.11 \%$ were intermediately resistant to it. 


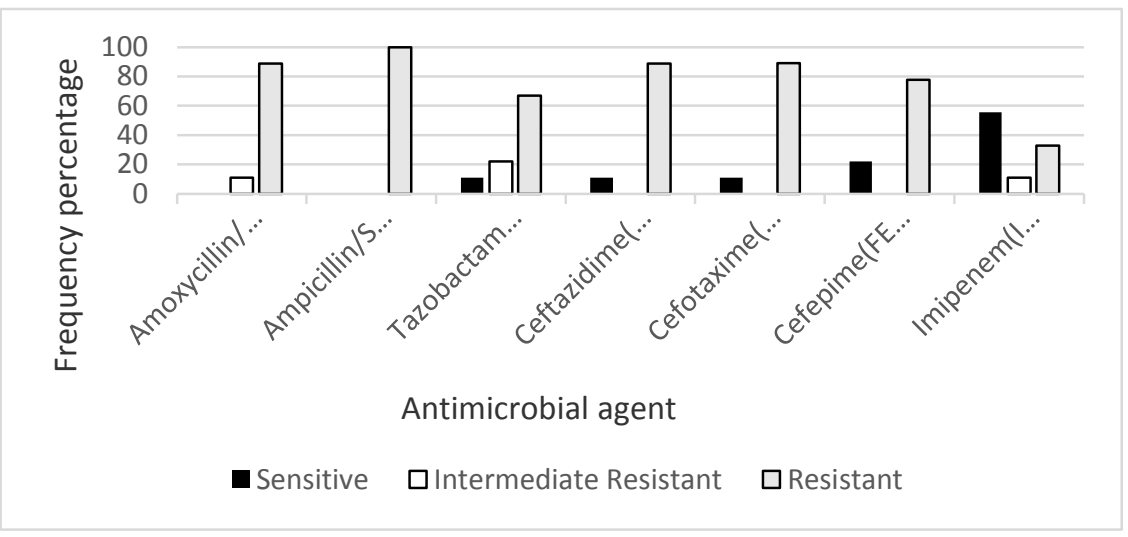

Fig.(4): Frequency percentages of different susceptibility profiles of bacterial isolates recovered from immunocompetent patients against some antimicrobial agents inhibiting bacterial cell wall synthesis

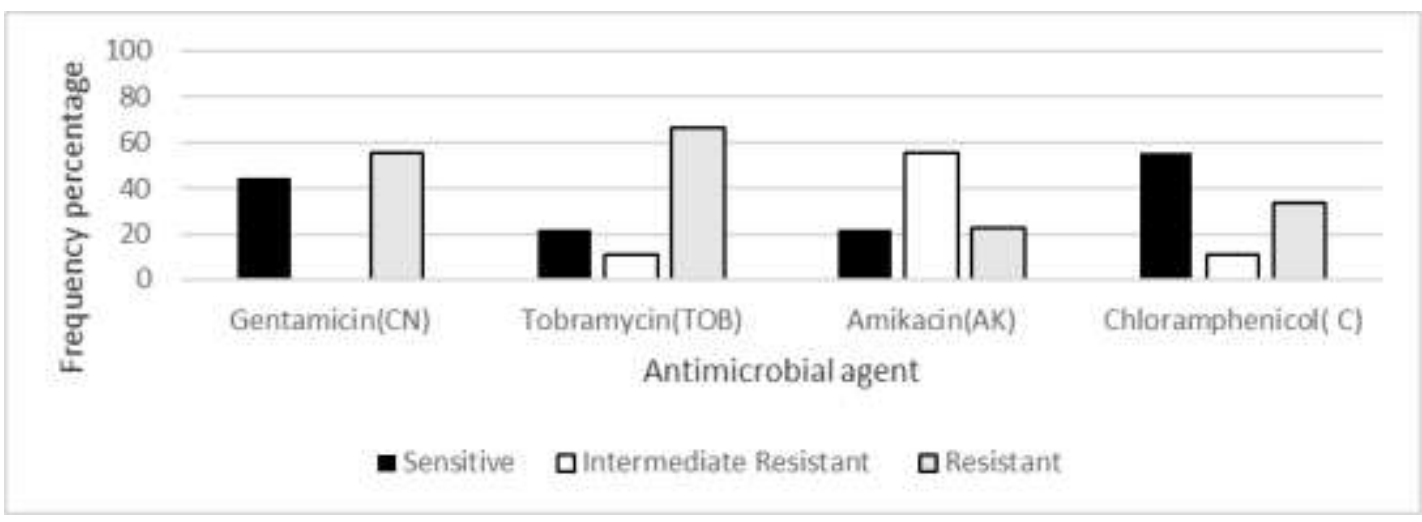

Fig. (5): Frequency percentages of different susceptibility profiles of bacterial isolates recovered from immunocompetent patients against some antimicrobial agents inhibiting protein synthesis in bacteria

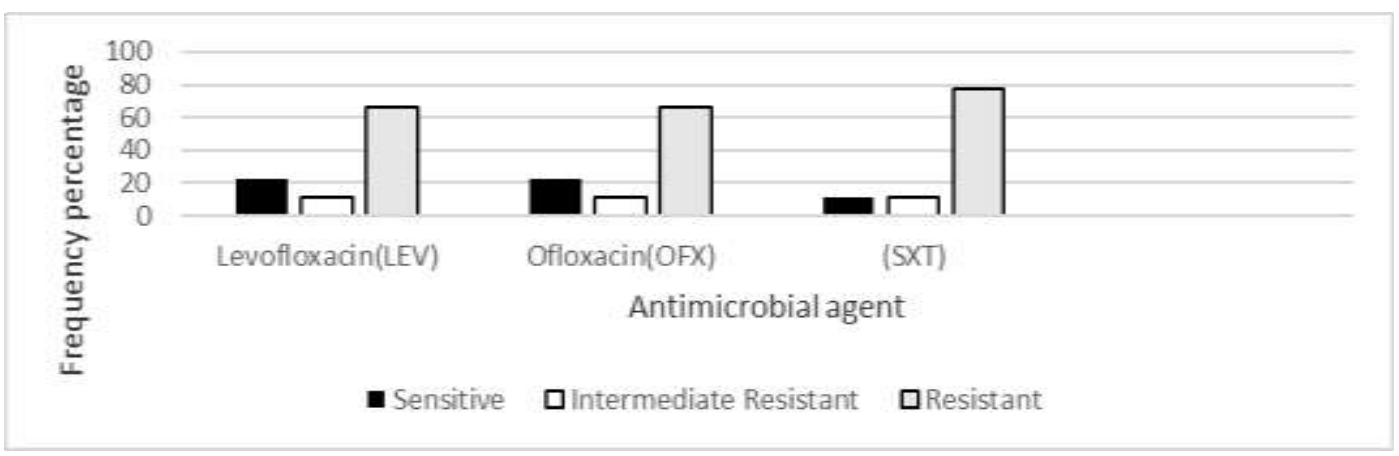

Fig.(6): Frequency percentages of different susceptibility profiles (sensitive, intermediate resistant and resistant) of bacterial isolates recovered from immunocompetent patients against some antimicrobial agents inhibiting nucleic and folic acids syntheses . 


\section{Activity profiles of lipase and protease enzymes of selected bacterial isolates recovered from patients:}

Lipase and protease enzymatic activities were determined for some tested organisms. The antimicrobial susceptibility testing of these isolates against some antimicrobial agents was also previously determined. The summarized data for lipase and protease enzymatic activities, resistance prevalence of each test organism against different tested antimicrobial agents are presented in table 3. It was clear that there was relationship between some virulence factors of tested bacterial isolates and severity of infection protease enzymatic activity.

Table (3): Profiles of lipase and protease enzymatic activities, resistance prevalence against different tested antimicrobial agents bacterial isolates recovered from different patients

\begin{tabular}{|c|c|c|c|c|}
\hline Patient's no. & Recovered isolate & $\begin{array}{c}\text { Lipas } \\
\text { e }\end{array}$ & $\begin{array}{c}\text { Protea } \\
\text { se }\end{array}$ & $\begin{array}{c}\text { Resistance prevalence } \\
(\%)^{*}\end{array}$ \\
\hline 1 & E. coli & - & + & 78.6 \\
\hline 2 & E. coli & - & + & 50 \\
\hline 3 & E. coli & - & - & 64.3 \\
\hline 4 & E. coli & - & - & 14.3 \\
\hline 5 & E. coli & - & - & 71.4 \\
\hline 6 & E. coli & - & + & 35.7 \\
\hline 7 & K pneumoniae & + & - & 64.3 \\
\hline 8 & K. pneumoniae & + & - & 78.7 \\
\hline 9 & K. pneumoniae & - & - & 50 \\
\hline 10 & K. pneumoniae & - & - & 64.3 \\
\hline 11 & Pseudomonas sp. & + & + & 50 \\
\hline 12 & Pseudomonas sp. & + & + & 35.7 \\
\hline 13 & Pseudomonas sp. & + & - & 85.7 \\
\hline 14 & $\begin{array}{r}\text { Acinetobacter } \\
\text { baumannii }\end{array}$ & + & - & 100 \\
\hline 15 & $\begin{array}{r}\text { Acinetobacter } \\
\text { baumannii }\end{array}$ & + & - & 71.4 \\
\hline 16 & K. pneumoniae & - & - & 85.7 \\
\hline 17 & K. pneumoniae & - & - & 64.3 \\
\hline 18 & $K$.pneumoniae & - & - & 64.3 \\
\hline 19 & K.pneumoniae & - & - & 71.4 \\
\hline 20 & E. coli & - & + & 78.6 \\
\hline 21 & E. coli & - & - & 78.6 \\
\hline 22 & E. coli & - & - & 50 \\
\hline 23 & $\begin{array}{r}\text { Acinetobacter } \\
\text { baumannii }\end{array}$ & - & - & 71.4 \\
\hline 24 & Pseudomonas sp. & + & + & 71.4 \\
\hline
\end{tabular}


${ }^{(*)}$ represents the organism resistance before exposure to gamma radiation to some antimicrobial agents relative to the total tested antimicrobial agents and expressed as percentage

\section{Discussion}

Bloodstream infection (BSI) continues to be a life threatening condition. Juan $\boldsymbol{e t}$ al., 2013 reported that prevalence of bacteremia for all neutropenia and fever events was $24.3 \%$ and Gram-negative bacteria were predominant $(65 \%)$, the ratio of isolates of gram negative and gram positive was 2.2 . The most frequently isolated bacteria were Pseudomonas sp. (21.6\%).

Many studies associate bloodstream infection in cancer patients with Gram-negative bacteria. Amongst Gram-negative organisms, Escherichia coli was the most common isolate; in immunocompromised patients, twice as many BSIs were caused by Pseudomonas aeruginosa and Enterobacter cloacae Bos et al., 2013.

In this study for feverish patients the prevalence of $E$. coli was $41 \%$ followed by Klebsiella pneumoniae $23 \%$ then Pseudomonas sp. 27\%, while for other feverish patients Klebsiella pneumoniae prevalence was $41 \%$ followed by E. coli $38 \%$ then Pseudomonas sp. 14\%. Neutropenic patients with bacteremia, in particular those with gram-negative bacteremia, appear to have a relatively high risk of complications and lethal outcome which is clearly higher than the risk of adverse outcomes associated with unexplained fever Engel et al., 2005.

Annette $\boldsymbol{e t}$ al., 2014 reported that Granzymes are serine proteases released by cytotoxic lymphocytes and induce cell death in virus-infected cells and tumor cells. However, granzymes also exist extracellularly in the blood circulation of patients with autoimmune diseases and infections and may contribute to inflammation. Human granzyme K (GrK) binds to Gramnegative bacteria and to lipopolysaccharide (LPS), a Gram negative bacterial cell wall component. In conclusion, GrK modulates the innate immune response against LPS and Gram-negative bacteria and may contribute to the pathogenesis of diseases associated with a local or systemic bacterial infection. Bloodstream infections (BSIs) and antimicrobial resistance (AMR) are worldwide health care problems causing substantial patient morbidity and mortality. Tamer et al., 2011 reported that Gram-negative bacteria accounted for $61.7 \%$ of total pathogens isolated from blood cultures. One major concern associated with gram-negative pathogens is the emergence of (extended-spectrum betalactamase) ESBL-producing strains. These strains are resistant to all betalactam antimicrobial agents except cephamycins and carbapenems. Isolation of multidrug-resistant ESBL-producing $K$. pneumoniae strains has been described, especially in ICUs Rebuck $\boldsymbol{e t} \boldsymbol{a l}$, 2000. Increased rates of multidrug-resistant Gramnegative strains have been highlighted among Enterobacteriaceae and nonfermenting Gram-negative rods, despite discontinuation of fluoroquinolone-based antibacterial prophylaxis for neutropenic patients. In addition, antimicrobial resistance and/or the 
inadequacy of empirical antibiotic treatment have been frequently linked to a worse outcome in cancer patients with bloodstream infections caused by Gram-negative isolates Trecarichi and Tumbarello, 2014.

In this study, 15 samples were selected for antimicrobial susceptibility test. The frequency percentages of resistant and susceptible bacterial isolates against different antimicrobial agents which are classified according to mechanism of action are presented in .It was clear that, the highest resistance frequency percentage for $\beta$ - lactam antibiotics was observed with: Ampicillin/Sulbactam (SAM) 80\%, Amoxicillin/Clavulanic acid (AMC) 66.66\%, Cefotaxime (CTX) 60\%, then Cefepime (FEP) 46.66\%, Ceftazidime (CAZ) 46.66\%, Tazobactam/Piperacillin (TZP) 40\%. On the other hand, Imipenem (IPM) showed no resistant phenotype for the tested isolates but intermediate resistant reaction $26.66 \%$. Some other antibiotics play an important role in the inhibition of protein synthesis, showed that most of the tested strains were resistant to Gentamycin $(\mathrm{CN})$ and Tobramycin (TOB) $73.33 \%$ followed by $33.33 \%$ were resistant to Amikacin (AK), all previous antibiotics belonged to aminoglycosides group. Meanwhile, resistance to Chloramphenicol (C) was represented by $40 \%$. In case of antibiotics which act on inhibition of nucleic acid synthesis, the results showed a high percentage of resistance among the isolated gram negative bacilli against Levofloxacin (LEV) $73.33 \%$ then Ofloxacin (OFX) 66.66\%. Sulphamethoxazole/Trimethoprim (SXT) as a type of antibiotic acts on inhibition of folic acid synthesis $80 \%$ of the isolates were resistant and $20 \%$ were intermediately resistant to it . Also, there was significant p-value $=\mathbf{0 . 0 0 8 6}, \mathbf{0 . 0 1 9 8}$ and $\mathbf{0 . 0 5}$ for AMC in E. coli strains, for TOB in Pseudomonas strains and SAM in Acinitobacter strains respectively.

Among the bacterial isolates recovered from other patients, Nine (9) isolates were selected for antimicrobial susceptibility test. With 7 antibiotics acting on the inhibition of cell wall synthesis, it was clear that the highest percentage of antibiotic resistance of nine bacterial strains was against Ampicillin/Sulbactam (SAM) 100\%, Amoxicillin/Clavulanic acid (AMC) 88.88\%, Cefotaxime (CTX) 88.88\%, Ceftazidime (CAZ) $88.88 \%$ then Cefepime (FEP) $77.77 \%$, Tazobactam/Piperacillin (TZP) $66.66 \%$, finally Imipenem (IPM) showed resistant reaction 33.33\%. Some other antibiotics play important role in the inhibition of protein synthesis. It was clear that most of the isolates were resistant to Tobramycin (TOB) 66.66\%, Gentamycin (CN) 55.55\% and 22.22\% were resistant to Amikacin (AK), all previous antibiotics belonged to aminoglycosides group. Resistance to Chloramphenicol (C) was represented by $33.33 \%$. In case of antibiotics which act on inhibition of nucleic acid synthesis, the results showed a high percentage of resistance among the tested strains against Levofloxacin (LEV) and Ofloxacin (OFX) 66.66\%. With Sulphamethoxazole/Trimethoprim (SXT) $77.77 \%$ of the treated isolates were resistant and $11.11 \%$ were intermediately resistant to it.

Extracellular or surface localization of virulence determinants is an important attribute of pathogenic microorganisms. The past decade has seen significant research advances in defining the steps and identifying the necessary machinery for protein secretion from 
bacterial cells Stephen et al., 2004. Correlations were clear within bacterial isolates recovered from leukopenic patients as For E. coli $(n=6)$, isolates recovered from patients howed high protease enzymatic activity and antimicrobial resistance reaching $78.6 \%$.

While those without protease activity were isolated from patients with antimicrobial resistance reaching $71.4 \%$. For Klebsiella pneumoniae $(\mathrm{n}=4)$, isolates recovered also showed lipase enzymatic activity with antimicrobial resistance reaching 78.7\%. For Pseudomonas species, showed protease and lipase activities. For Pseudomonas species $(\mathrm{n}=3)$, isolates recovered showed protease and lipase enzymatic activities.. For Acinitobacter baumannii ( $\mathrm{n}=2)$, isolates recovered from patients showed lipase enzymatic activity with $\mathbf{p}$-value $=\mathbf{0 . 0 4}$ and antimicrobial resistance reaching $78.7 \%$.While, for bacterial isolates recovered from other patients, there was no clear correlation, except for no.24Pseudomonas fluorescence there was positive lipase and protease activity.

\section{REFERENCES}

Annette C. Wensink, Vera Kemp, Job Fermie, M. Isabel García Laorden, Tom van der Poll, C. Erik Hack, and Niels Bovenschen, Granzyme K (2014): synergistically potentiates LPS-induced cytokine responses in human monocytes, PNAS,April 22, 2014,vol. 111, no. 16, 5974-5979

Bauer, A. W., M. Kirby, J. C. Sherris, and M. Turck,( 1966): Antibiotic susceptibility testing by a standardized single disk method. Am. J. Clin. Pathol. , 45:493-4\%.

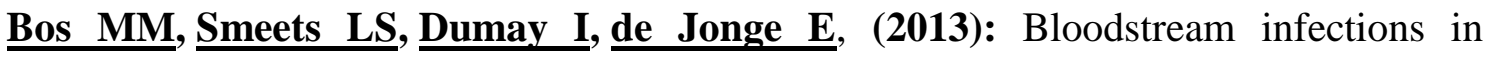
patients with or without cancer in a large community hospital, Infection (Oct;41(5):949-58.

Collee, J.G., R.S. Miles and B. Watt, (1996):. Tests for the identification of Bacteria. In: Collee, J.G., A.G. Fraser, B.P. Marimon and A. Simmons (Eds.), Mackie and McCartney Practical Medical Microbiology. 14th Edn., Churchill Livingstone, London, pp: 131-149.

De Martel C, Franceschi S.( 2009):, Infections and cancer: established associations and new hypotheses, Crit Rev Oncol Hematol. Jun;70(3):183- 
Dominique M Vandijck, Eric A Hoste, Stijn I Blot, Pieter O Depuydt, Renaat A Peleman and Johan M Decruyenaere(2007):, Dynamics of C-reactive protein and white blood cell count in critically ill patients with nosocomial Gram positive vs. Gram negative bacteremia: a historical cohort study, BMC Infectious Diseases, 7:106 doi:10.1186/1471-2334-7-106.

Engel, S.A. Knoll, P. Kern, W.V. Kern, (2005): Interleukin-8 Serum Levels at Fever Onset in Patients with Neutropenia Predict Early Medical Complications, Infection; 33: 380-382.

H. Aalto . A. Takala - H. Kautiainen - H. Repo, (2004) : Laboratory markers of systemic inflammation as predictors of bloodstream infection in acutely ill patients admitted to hospital in medical emergency, Eur J Clin Microbiol Infect Dis 23: 699-704

Hülya Sungurtekin MD, Ugur Sungurtekin MD, Canan Balci MD (2006):, Circulating complement (C3 and C4) for differentiation of SIRS from sepsis, Advances in Therapy, November/December, Volume 23, Issue 6, pp 893-901.

Jørgensen,P.M. \& León-Yánez,S. (eds.) (1999): Catalogue of the Vascular Plants of Ecuador. Monographs in Systematic Botany from the Missouri Botanical Garden. 75: i--viii, 1-1182.

Juan C.Reyes, M.M.Aguilera, A.C.Zamora and J.G.Meza(2013):, Frequency of risk factors for bacteremia in children with cancer, neutropenia and fever in a tertiary level hospital in western Mexico, Bol Med Innfant Mex,;70(4):303308.

M. von Lilienfeld-Toal . M. P. Dietrich .A. Glasmacher . L. Lehmann . P. Breig • C. Hahn .I. G. H. Schmidt-Wolf . G. Marklein . S. Schroeder .F. Stuber (2004) : , Markers of bacteremia in febrile neutropenic patients with hematological malignancies: procalcitonin and IL-6 are more reliable than Creactive protein, Eur J Clin Microbiol Infect Dis 23: 539-544.

MATSEN, J. M., A. L. BARRY (1974): Manual of Clinical Microbiology. 2nd ed. American Society for Microbiology, Washington, DC. pp. 418-427.

Michael J. Pecaut, Farnaz P. Baqai, and Daila S. Gridley,( 2014): Impact of totalbody irradiation on the response to a live bacterial challenge, July, Vol. 90, No. 7 , Pages 515-526 
Miriam Diepold, Peter Noellke, Ulrich Duffner,(2008): Udo Kontnyand Reinhard Berner,Performance of Interleukin-6 and Interleukin-8 serum levels in pediatric oncology patients with neutropenia and fever for the assessment of low-risk, BMC Infectious Diseases, 8:28.

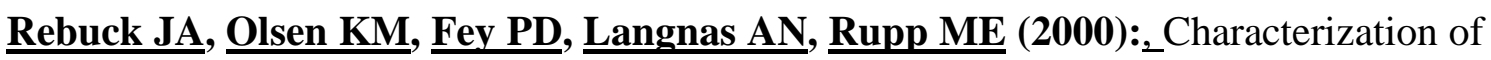
an outbreak due to extended-spectrum beta-lactamase-producing Klebsiella pneumoniae in a pediatric intensive care unit transplant population, Clin Infect Dis. Dec;31(6):1368-72. .

Ryuzo Abe, Shigeto Oda, Tomohito Sadahiro, Masataka Nakamura, Yo Hirayama, Yoshihisa Tateishi, Koichiro Shinozaki and Hiroyuki Hirasawa(2010): , Gram-negative bacteremia induces greater magnitude of inflammatory response than Gram-positive bacteremia, Critical Care, 14:R27 Volume 14, Issue 2

Stéphane Hugonnet, Hugo Sax, Philippe Eggimann, Jean-Claude Chevrolet and Didier Pittet,(2004): Nosocomial Bloodstream Infection and Clinical Sepsis Emerging Infectious Diseases Vol. 10, No. 1, January,75-81.

Stephen Lory,(1998): Secretion of proteins and assembly of bacterial surface organelles: shared pathways of extracellular protein targeting, $\underline{\text { Current Opinion }}$ in Microbiology, Volume 1, Issue 1, February, Pages 27-35.

Tamer Saied, Amany Elkholy, Soad F. Hafez, Hadia Basim, Momtaz O. Wasfy, Waleed El-Shoubary, Ahmed Samir,( 2011): Guillermo Pimentel and Maha Talaat, MPH, Antimicrobial resistance in pathogens causing nosocomial bloodstream infections in university hospitals in Egypt, Association for Professionals in Infection Control and Epidemiology,Inc.,39,15.

Thaler, J.O., Duvic, B., Givaudan, A., Boemare, N., (1998): Isolation and entomotoxic properties of the Xenorhabdus nematophilus F1 lecithinase. Appl. Environ.Microbiol. 64, 2367-2373.

Trecarichi EM, Tumbarello M.,(2014): Antimicrobial-resistant Gram-negative bacteria in febrile neutropenic patients with cancer: current epidemiology and clinical impact, Curr Opin Infect Dis. Apr;27(2):200-10

Young, L. S. (2008): “Bacteremia. Merck Manual Home Health Handbook.” Updated 
Accessed September (2012).

Yoshihiko Sawa, Takeshi Ueki, Minoru Hata, Kana Iwasawa, Eichi Tsuruga, Hiroshi Kojima, Hiroyuki Ishikawa, and Shigemitsu Yoshida,(2008): LPSinduced IL-6, IL-8, VCAM-1, and ICAM-1 Expression in Human Lymphatic Endothelium, Journal of Histochemistry \& Cytochemistry,, Volume 56(2): 97109 , 


$$
\text { الملخص العربي }
$$

لمحه عن النشاط الانزيمي لليبيز و البروتيز و مقاومه مضادات الميكروبات لبعض العزلات

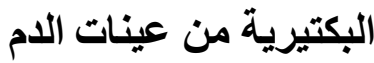

$$
\begin{aligned}
& \text { للسادة الدكاترة }
\end{aligned}
$$

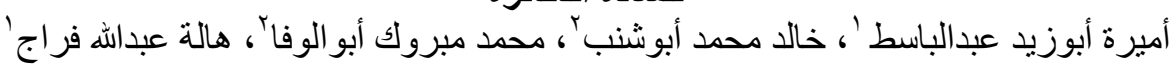<smiles>[CH]</smiles>

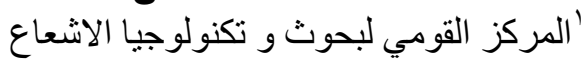

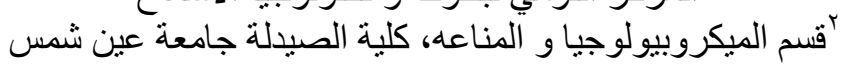

ان عدوي مجري الدم تستمر لتكون حاله تهدد الحياه. رد فعل العائل لهذه العدوي يختلف عن الدلالات الطبية

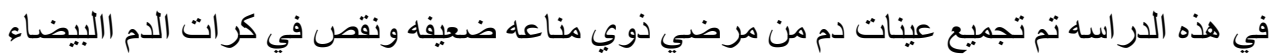

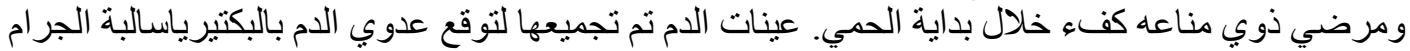

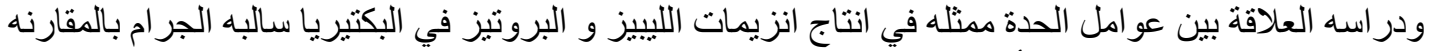

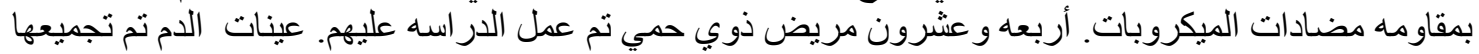

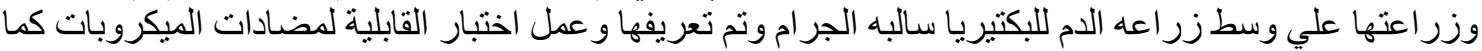

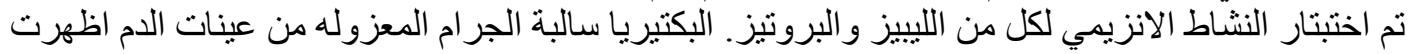

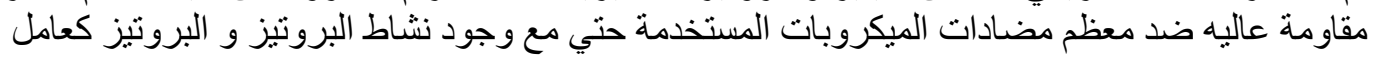

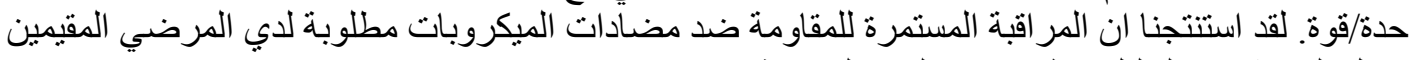
داخل المستشفيات لتقليل خطر عدوي الدم و المقاومة. 